\title{
MULTI-AXIAL SELF-ORGANISATION PROPERTIES OF MOUSE EMBRYONIC STEM CELLS INTO GASTRULOIDS
}

Leonardo Beccari $^{1, *}$, Naomi Moris ${ }^{2, *}$, Mehmet Girgin ${ }^{3, *}$, David A. Turner ${ }^{2}$, Peter Baillie-Johnson ${ }^{2,4}$, Anne-Catherine Cossy ${ }^{3}$, Matthias P. Lutolf ${ }^{3}$, Denis Duboule ${ }^{1,3, \text { I }}$ and Alfonso Martinez Arias ${ }^{2,1}$.

${ }^{1}$ Department of Genetics and Evolution, University of Geneva, Geneva, Switzerland.

${ }^{2}$ Department of Genetics, University of Cambridge, Cambridge, UK.

${ }^{3}$ School of Life Sciences, Federal Institute of Technology EPFL, Lausanne, Switzerland.

${ }^{4}$ Current address: Wellcome Trust-Medical Research Council Cambridge Stem Cell Institute, University of Cambridge, UK.

*Shared first authorship

"Shared last authorship

Correspondence to:

Alfonso Martinez-Arias: ama11@hermes.cam.ac.uk

Denis Duboule:Denis.Duboule@epfl.ch 
The emergence of multiple axes is an essential element in the establishment of the mammalian body plan. This process takes place shortly after implantation of the embryo within the uterus and relies on the activity of Gene Regulatory Networks (GRNs) that coordinate transcription in space and time. While genetic approaches have revealed important aspects of these processes ${ }^{1}$, a mechanistic understanding is hampered by the poor experimental accessibility of early post-implantation stages. Here we show that small aggregates of murine Embryonic Stem cells (ESC) stimulated to undergo gastrulation-like events and elongation in vitro, are capable of organising a post-occipital pattern of neural, mesodermal and endodermal derivatives that mimic the embryonic spatial and temporal gene expression. The establishment of the three major body axes in such 'gastruloids' 2,3 suggests that the mechanisms involved are interdependent. Specifically, gastruloids display the hallmarks of axial gene regulatory systems as exemplified by the implementation of Hox collinear transcriptional patterns along an extending anteriorposterior axis. These results reveal an unanticipated self-organising capacity for aggregated ESC and suggest that gastruloids may be used as a complementary system to study early developmental events in the mammalian embryo.

Recent work on stem cell derived organoids has revealed a surprising autonomy in the development of particular tissues and organs ${ }^{4,5}$. When $c a$. 250 ESCs are aggregated, given a pulse of the Wnt agonist CHIR99021 (Chi) between 48 and 72h of culture, and returned to N2B27 medium (Fig. 1a), a pole of T/Brachyury (Bra) expression, resembling the elongating embryonic tail bud emerges reproducibly ${ }^{6}$ (Fig. 1b, Extended data Fig. 1). The aggregates keep elongating up to $120 \mathrm{~h}$ after aggregation (AA), when they display a 'rostral' cell-dense region and a polar extension towards a 'caudal' extremity reaching up to $500 \mu \mathrm{m}$ in size (Fig. 1b). Shaking the culture allows to reach 850 1000 micron in length at $168 \mathrm{hrs}$ AA (Fig. 1c,d). At these late stages, a Gata6-positive domain is detected at the opposite side of a $B r a$ and $C d x 2$ expressing region, likely corresponding to the cardiac crescent, which delimits the embryonic post-occipital region ${ }^{7}$ (Fig. 1b-d, Extended data Fig.1, Supplementary Movies 1 and 2). In contrast, Sox1/Sox2 positive cells localised centrally, with the exception of the rostral-most portion (Fig. 1c, d).

To characterize the transcriptional programmes of these gastruloids, we carried out RNAseq on duplicated pools and compared their profiles with those of developing mouse embryos from E6.5 to E9.5. Since gastruloids display hallmarks of post-occipital embryos ${ }^{6}$ (Fig.1b-d) we excluded the anterior portion of E7.5-E9.5 embryos (Fig. 1e, top). Principal Component Analysis (PCA) showed reproducibility between samples and a clear clustering along $\mathrm{PC} 1$ corresponding to the temporal 
order of samples (Fig. 1e), while embryo samples segregated from gastruloids in PC2 only. The main (top 100) clustering determinants of gastruloid samples included several pluripotency-related genes, epiblast markers and genes involved in gastrulation, as well as Hox genes and other transcription factors such as Cdx1/2, Meis1/2, Meox1, Bra, and Gata4 (Figure 2a). These genes are normally expressed in post-occipital structures of the developing mouse embryo. 25 out of 100 of these PCA determinants were identified independently in both gastruloids and embryos temporal series, (Figure 2a, red-labelled genes) supporting the idea that gastruloids and embryos elongate by implementing similar transcriptional programs. The analysis of specific genes associated with particular developmental landmarks confirmed this point (Fig. 2b, Extended data Fig. 2b). For instance genes associated with gastrulation like Mixl1, Eomes, Gsc or Chrd were transiently and orderly transcribed at around 48h AA (Fig. 2b and Extended data Fig. 2), suggesting that at this stage the gastruloids transcriptome resembles that of mouse epiblast at the onset of gastrulation. By $72 \mathrm{~h}$ AA, we observed an increase in the complexity of gene expression profiles, with the appearance of markers for different embryonic lineages including mesendoderm and neuroectoderm and the transcription of Hox gene clusters (Extended data Fig. 2 and Fig. 2a, b; see below). Genes associated either with extraembryonic structures or with anterior neural plate derivatives were not (or poorly) expressed in gastruloids (Fig. 2b, Supplementary information Files 1 and 2),

PCA analysis using single gastruloids revealed a robust clustering for any developmental stage assessed and with the pooled RNAseq datasets (Extended data Fig. 2c) showing that the population of gastruloids was rather homogenous at the time points analysed and hence that the pooled RNAseq datasets reflected the transcription status of single gastruloids. Gastruloids transcriptome analyses revealed mRNAs normally associated with neural, endodermal and mesodermal derivatives, including paraxial, cardiac, intermediate and hematopoietic progenitors as well as neural crest (e.g. ${ }^{8,9}$ ) (Fig. 2b; Extended data Figs. 2b and 3). We also observed an anteroposterior pattern of differentiation along these lineages, reminiscent to what occurs in the embryo. For example, the sequential expression of Bra, Msgn1, Meoxl/Tcf15 recapitulates the spatiotemporal differentiation pattern of paraxial mesoderm (Extended data Fig. 3a, b). In neural tissue, while Hes 5 and Dll 1 were strongly expressed during gastruloid extension, the density of terminally differentiating Phox2a/Mnxl positive cells formed an apparent anterior to posterior gradient, almost completely absent from the posterior-most aspect (Extended data Fig. 3c, d). These ordered patterns of gene expression nevertheless did not correlate with any precise morphogenesis. Neural markers (Sox1, Sox 2, Lnfg) were expressed in a continuous domain, yet without forming a proper neural tubelike structure (Extended data Figs. 3d and 4a-c; Fig. 3) even though sporadic tubular structures were 
scored along this domain (Extended data Fig. 4a, white arrowheads, c, right panel). Also, clumps of cells positive for either SOX1 and OLIG2, SOX1 and PAX3 or SOX1 and PAX7, indicative of dorsal and ventral neural tube progenitors, were observed (Extended data Fig. 4d), yet without a clear segregation along the dorso-ventral extension of the SOX1 domain. Similarly, Tcf15 expressing cells did not condense into somites (Extended data Fig. 3b).

The analysis of different endodermal markers revealed temporal dynamics also reminiscent of the embryonic situation ${ }^{10}$ (Extended data Figs. 3e, f and 4e-f). Gsc and $C d x 2$ transcripts, markers of definitive endoderm ${ }^{11,12}$ were upregulated soon $(72 \mathrm{~h} \mathrm{AA})$ after Chiron induction, followed by Cer1 (96-120h AA) and by Sorcs2, Pax9 or Shh subsequently (120h-144h AA). All endoderm expressed genes assayed (Sox17, Sorcs2, Nedd9, Pyy, Cdx2 and Shh) were active in the ventral-like domain of gastruloids (Fig. 3, Extended data Figs. 3f, 4e-f), resembling the embryo situation. Of note, $C d x 2$ transcripts were confined to the posterior most gastruloid endoderm (Fig. 1d, Extended data Fig. 4f), in agreement with this gene specifying the hindgut domain. In a majority of cases, gutendoderm progenitors appeared as a continuous tubular structure (Extended data Fig. 4a, e-f; red arrowheads), often spanning the entire antero-posterior extension, reminiscent of an embryonic digestive tract.

This unanticipated level of organization and capacity to self-organize an integrated axial system reminiscent of the embryo was further explored by assessing the expression of genes associated with the developing embryonic axes (Fig. 3). Wnt3a and Cyp26al transcripts were scored at the caudal extremity of gastruloids similar to Bra and Cdx2 (Fig. 1c, d; Extended data Figs. 3a, d and 4a, d, Extended data Fig. 5a), complementary to the localization of Raldh2 mRNAs further supporting the existence of an antero-posterior axis. On the other hand, Lnfg, Sox1 and Sox2 were transcribed in a central and dorsal domain at 144h AA (Figs. 1b, 3b and Extended data Figs. 3a and 4a), complementary to the ventrally located intestinal tract markers (Figs. 3b, Extended data Figs. 3d and 4c, d, Extended data Fig. 5b). Additional signs of multi-axial organisation were provided by the expression of mesoderm specific genes Osr1, Pecam, Meox1 and Pax2 in a medio-lateral symmetry flanking the centrally located Sox2 positive domain (Fig. 3c). Double staining of Sox 2 and Meox 1 (Fig. 3c, Extended data Fig. 5c) and cross-sections (Extended data Fig. 4b) confirmed the mediolateral and dorso-ventral distribution of neural and mesodermal progenitors.

Nodal expression was found confined to a small and compact region on the ventral most posterior aspect at 120h AA (Extended data Fig. 6 and 7). These cells displayed high levels of Ecadherin and a dense phalloidin staining (Extended data Fig. 6a, b) suggestive of a node-like identity, a hypothesis supported by the presence of Nodal mRNAs in a domain comparable to that of 
Goosecoid, Bra and Chordin at 96h AA (Extended data Fig. 6c,d). Time course analysis of Chrd and Nodal indicated that such putative node-like cells were detected at $96 \mathrm{~h}$ and persisted until $144 \mathrm{~h}$ at least (Extended data Fig. 6d). Nodal mRNAs in these cells nevertheless rapidly decreased and were almost undetectable at $144 \mathrm{~h}$ AA. Despite these evidences for a node-like structure, we did not observe any notochord derivatives, which normally originate from the node, raising questions as to whether this putative structure may excert an organizing activity reminiscent to that of its embryonic counterpart. The downregulation of Nodal in presumptive node-like cells at 120h AA coincided with patches of Nodal-expressing cells along the posterior half of extending gastruloids, often distributed in an asymmetric manner (Fig. 3d, Extended data Fig 6d, e). This pattern was maintained at 144h AA (extended data Fig. 6d) but was not observed with Meox1, which was predominantly expressed on both sides (Fig. 3d, e). Accordingly, the Nodal target gene Cerberus was also expressed asymmetrically at both $120 \mathrm{~h} \mathrm{AA}$ and $144 \mathrm{~h} \mathrm{AA} \mathrm{(Extended} \mathrm{data} \mathrm{Fig.} \mathrm{6f).} \mathrm{Altogether} \mathrm{these} \mathrm{data} \mathrm{suggest}$ that besides the establishment of a medio-lateral axis, gastruloids may implement the start of a leftright asymmetry,

The formation and patterning of post-occipital embryonic territories is tightly linked to the sequential activation of the 39 Hox gene, which are clustered at four distinct genomic loci in mammals. As Hox genes appeared differentially regulated in the RNAseq time-course (Fig. 2a, b, Extended data Fig. 2a), we assessed whether their sequential activation in time and space ${ }^{13}$ was recapitulated too. A pooled PCA analysis considering exclusively Hox genes transcripts, revealed robust clustering along the time axis ( $81 \%$ variance) and a close correspondence with the dynamic activation of these genes in embryo (Fig. 4a, Extended data Fig. 8a-c). The variability in Hox RNAs content amongst gastruloids was then evaluated using ten individual specimens from three different stages (Extended data Fig. 9a). Gastruloids from the same time-point tightly clustered together solely based on their Hox transcripts. Transcript profiles over Hox clusters revealed signs of collinear activation, the hallmark of this gene family ${ }^{14}$. In E6.5 embryo, some Hoxa and Hoxd genes are expressed before gastrulation in extraembryonic tissues ${ }^{15}$ (Extended data Fig. 8a). From E7.8 to E9.5, Hox genes start to be transcribed in an order which reflect their 3' to 5' position within each cluster (Extended data Fig. 8a, b). The RNAseq profiling revealed an activation dynamic comparable to that observed in embryo (Fig. 2a and Extended data Fig. 8c). For instance while Hoxa RNAs were not detected until $48 \mathrm{~h}$ AA, Hoxal to Hoxa3 expressions were robust at $72 \mathrm{~h}$, followed by sustained transcription of Hoxa5, Hoxa 7 and Hoxa9 at $96 \mathrm{~h}$ to $120 \mathrm{~h}$. Hoxal0 and Hoxal1 RNAs appeared at 144h AA, at the same time Hoxa1, Hoxa2 and Hoxa3 transcripts started to disappear (Fig. 4b, Extended data Fig. 8c). Similar dynamics were observed for Hoxd genes (Extended data Fig. 8c), which were activated in a sequence starting from $72 \mathrm{~h}$ AA until 168h AA (Extended data Fig. 8c-e). 
The early transcription of 5' Hoxa/Hoxd genes (Extended data Fig. 8a-b) was not observed in gastruloids (Extended data Fig. 9c, d and Fig. 4b), in agreement with the absence of extraembryonic derivatives.

Comparable profiles were also scored when single organoids were examined (Extended data Fig. 9a, b), again revealing the high reproducibility of this activation process. In the embryo this temporal activation is paralleled by a collinear distribution of transcripts in space ${ }^{14}$. Likewise, Hoxa4/Hoxd4 displayed an AP boundary close to the anterior aspect of the gastruloid, whereas Hoxa9/Hoxd9, Hoxa11/Hoxd11 and Hoxd13 showed successively more posterior boundaries (Fig. 4c, Extended data Fig. 9c). Notably, Hoxd13 transcripts appeared in a population of cells located centrally at the posterior extremity, resembling its normal expression in the embryonic cloacal area (Fig. 4c). Hoxa13 expression was also detected at 168h AA in the posterior aspect, yet rarely $(1 / 20)$, in agreement with the low transcript levels detected in the pooled RNAseq analysis (Extended data Fig. 9c). Double staining for Hoxd4 and either Sox 2 or Meox1 revealed expression of Hox genes in both neural and mesodermal derivatives (Fig. 4d, Extended data Fig. 5d, e). The implementation in space and time of the Hox gene network confirmed the surprisingly high level of organisation in the processing of gene regulatory networks, in particular without any extraembryonic component (see ref. $\left.^{16}\right)$.

We tested the ability of several induced pluripotent stem cells (iPSC) lines to produce gastruloids (Extended data Fig. 10) and a similar elongation process was observed in one of them. iPSCs can thus generate gastruloids. However, these gastruloids showed a reduced elongation rate, particularly between $48 \mathrm{~h}$ and $96 \mathrm{~h}$ (extended data Fig. 10a, b). The expression dynamics of Bra in these iPSC gastruloids was nevertheless similar to their ESC counterparts (Extended data Fig. 11c, d). The neural markers Sox1 and Sox2 as well as $C d x 2$ were also expressed as in ESC-derived gastruloids (Extended data Fig. 10d, compare with Fig. 1b, c). Furthermore, iPSC gastruloids implemented Hoxd temporal and spatial collinear expressions, though with a delay in the expression onset and a spatial collinearity not as clearly organized as in ESC-derived gastruloids (Extended data Fig. 9d, e).

When compared to single tissue organoids (e.g. ${ }^{17,18}$ ), gastruloids exhibit an integrated structure, which seems to specify all major embryonic axes in a coordinated manner, thus complementing recent reports where stem cells were used to recapitulate morphological and transcriptional events of early blastocyst, yet not of subsequent embryonic stages ${ }^{19,16}$. However, the activation of tissue specific patterns of gene expression in our gastruloids was not paralleled by a clear internal organization of the corresponding embryonic tissue layers. This observation suggests 
that a direct causal relationship between transcriptional programs and early morphogenesis ought to be considered with caution. One potential reason for the low level of tissue organization in gastruloids may be the absence of mechanical interactions and constraints that characterize the developing embryonic context ${ }^{20}$. The remarkable autonomy in the patterns of gene expression reported here highlights the potential of gastruloids in the study of complex regulatory circuits, particularly during early post-implantation development and the emergence of body axes.

\section{Acknowledgements}

We thank members of the Duboule, Lutolf and Martinez-Arias laboratories for sharing material and discussions, J. Deschamps for DNA clones and D. Trono for the Oct4::GFP iPS cell line. We also thank the histology platform and the Gene Expression Core Facility (EPFL) as well as the genomics platform in Geneva University. This work was supported by funds from the BBSRC (No. BB/M023370/1 and BB/P003184/1 to AMA), an NC3Rs David Sainsbury Fellowship (No. NC/P001467/1 to D.A.T.), an Engineering and Physical Sciences Research Council (EPSRC) Studentship (to P.B.-J.), a Company of Biologists Development Travelling Fellowship (DEVTF-151210 to P.B.-J.), a Newnham College Constance Work Junior Research Fellowship (to NM), the Ecole Polytechnique Fédérale de Lausanne (D.D. and M.L.), the University of Geneva (D.D.), the Swiss National Research Fund (No. 310030B_138662 to D.D.) and the European Research Council grants ERC MOFDH (No. 250316 to A.M.A.), RegulHox (No 588029 to D.D.) and STEMCHIP (No 311422 to M.L.).

\section{Authors contributions:}

Conceived the study: AMA, DD, LB, MG, ML

Conducted experiments:, ACC, DT, LB, MG, MM, PBJ

Analyzed and interpret datasets: DD, NM, LB, MG, PBJ.

Wrote the Manuscript: AMA, DD, LB.

Corrected/Amended the MS: MG, ML, NM. 


\section{Legends to Figures}

Figure 1: Elongation of gastruloids. a. Schematic of the culture protocol. 200 to 300 ESCs were allowed to aggregate. The Wnt agonist CHIR99201 (Chi) was added between $48 \mathrm{~h}$ and $72 \mathrm{~h}$ after aggregation. Organoids were kept in suspension until 120h (grey rectangle) and transferred into shaking cultures until $168 \mathrm{~h}$. b. Three dimensional renderings and confocal sections of gastruloids at different times showing the elongation and expression of BRA, SOX2 and Gata6 ${ }^{\text {H2B-Venus }}$ (green). c-d. Three-dimensional rendering (c, left panel) and confocal sections (c, central and right panels and $\mathbf{d}$, zoom of tail region) of gastruloids at $168 \mathrm{~h}$, showing the localization of CDX2, SOX2, SOX1 and BRA proteins. Scale bar: $150 \mu \mathrm{m}$. For each time point analyzed, the results reported were scored in at least $80 \%$ of the cases $(n \geq 20)$. e. PCA analysis of RNAseq datasets using time-pooled gastruloids from $24 \mathrm{~h}$ to $168 \mathrm{~h}$ ( 2 replicates per time-point) and pooled mouse embryos at E6,5 (3 replicates), E7.8 (3 replicates), E8.5 (12-14 somites, 2 replicates) and E9.5 (ca. 24 somites, 2 replicates). For E7.8 embryos, the posterior half was used. For E8.5 and E9.5, the post-occipital embryonic domain was used. The dissected portion is colored in pale green. All autosomal genes were considered for this analysis. Principal Component 1 (PC1) shows a strong temporal component while PC2 discriminates between gastruloids or embryonic samples.

Figure 2. Temporal patterns of gene expression in gastruloids. a. PCA of either pooled gastruloids during temporal progression from $24 \mathrm{~h}$ to $168 \mathrm{~h}$ (left), or murine embryos from E6.5 to E9.5 (right). The 100 top contributing genes to the first two principal components are overlaid, with those observed in both gastruloids and embryonic datasets shown in red text. b. Heatmap of scaled expression of genes associated with development of different embryonic structures in pooled gastruloids and embryos over time.

Figure 3: Multi-axial organization of gastruloids. a-c. Gene expression in gastruloids at 144h AA showing their axial organization. a. Wnt3a and Cyp26al expression (arrowhead) at the posterior end, where Raldh2 is not transcribed (empty arrowhead). Double FISH staining of Meox1 and Cyp26a1 (a, right-most panel) showing antero-posterior segregation of mesodermal precursors. b. Dorso-ventral (D-V) axis revealed by the ventral expression of Shh and Krt18, and of Lnfg dorsally (empty arrowheads). Double FISH staining of Sox2 and Shh confirmed a dorso-ventral segregation, with Shh expressed exclusively in endoderm precursors (b, righ panel). C. Medio-lateral (M-L) axis of symmetry (dotted line) revealed by the bilateral expression of Meox1 and Pax2, complementary to the central distribution of Sox2 transcripts (empty arrowheads). For each gene, the proportion of gastruloids displaying the reported pattern is shown. Scale bar: $100 \mu \mathrm{m}$. d. 3D renderings of confocal 
stacks of $120 \mathrm{~h}$ gastruloids containing a $\mathrm{Nodal}^{Y F P}$ reporter gene, stained for SOX2 (white) and BRA (red) proteins and imaged from the dorsal (left) and ventral (right) orientations; insets details of the posterior region. Reporter gene expression within the Bra expressing domain on the ventral surface is suggestive of a node-like structure (middle panel; Extended Figure 6). Additional expression of Nodal as a bilaterally asymmetric cluster of cells (white open arrow) is reminiscent of the asymmetric Nodal expression in the embryo (middle panel). Right panel shows a posterior view of the $3 \mathrm{D}$ rendering. e. Bar graph showing the frequency distribution of asymmetric and symmetric expression of Nodal or Meoxl in 120h gastruloids (see Extended data Table 1 for more details). \#\#\# (observed versus expected frequency, based on the embryonic gene expression pattern): $p<0,0001$; **(observed versus expected frequency in asymmetric Nodal expression based on the frequency of Meoxl asymmetry in gastruloids).

Figure 4: Collinear Hox gene expression in gastruloids. a. PCA plot solely based on Hox transcripts datasets extracted from pooled gastruloid and embryonic data across time points. Replicate batches of organoids primarily cluster according to their age at collection. b. Transcript profiles over the HoxA cluster, using time-sequenced pooled gastruloids. A progressive wave of transcription through Hoxa genes is observed between the $72 \mathrm{~h}$ and $168 \mathrm{~h}$ time-points. c. In situ hybridizations of $168 \mathrm{~h}$ gastruloids using probes for various Hoxd genes. Expression becomes spatially restricted along the A-P axis along with the respective position of the genes in the cluster. For each gene, the proportion of gastruloids displaying the reported expression pattern is shown in the bottom right corner of the image, expressed as a fraction of the total number of gastruloids analyzed. Scale bar: $100 \mu \mathrm{m}$. d. Double FISH staining of Hoxd4 with Sox2 or Meox1 (respectively marking the neural and mesodermal precursors) showed that Hoxd4 expression colocalized with both markers, suggesting that gastruloid implement both neural and mesodermal Hoxd gene expression. Scale bar: $200 \mu \mathrm{m}$.

\section{Materials and Methods}

ES/iPS cells and gastruloid cultures: The culture conditions and a detailed protocol for ES/iPS cells culturing and gastruloid production can be found in Supplementary material and method and in the associated Protocol Exchange article.

Animal experimentation: wild-type CD1 mouse embryos were used for RNAseq experiments. All experiments were performed in agreement with the Swiss law on animal protection (LPA) under license number GE 81/14 (to D. Duboule). 
RNA extraction and RNAseq libraries preparation and sequencing can be found in supplementary information.

Libraries and qPCR analysis: Purified RNA from iPS cell derived gastruloids was retrotranscribed using the Promega GoScript retrotranscription kit. Quantitative PCR analysis of mRNA levels for different Hoxd genes, Bra and the housekeeping gene Hmbs was performed using the Syber select master mix for CFX (Thermofisher) kit according to manufacturer instruction and specific primers ${ }^{6,21}$. The Biorad CFX96 thermocycler was used. At least two technical (PCR) replicates and two biological replicates were analyzed per time-point after aggregation.

Probe cloning, in vitro transcription and in situ hybridization. Refer to Supplementary material and methods and to Extended data Tables 3 and 4.

Immunostaining and confocal microscopy. Refer to Supplementary material and methods and to Extended data Table 5.

Data availability statement. All RNAseq datasets produced in this study are publicly available in the Gene Expression Omnibus (GEO) database under \#GSE10622. All the scripts used for the analyzis of the RNAseq data are freely available upon request.

\section{Extended data figure legends}

Extended data Figure 1. a-c. Gastruloids produced using Gata $6^{\text {H2B-Venus }}$ mESCs treated with a pulse of the GSK3 inhibitor Chiron between $48 \mathrm{~h}$ and $72 \mathrm{~h} \mathrm{AA}$ and fixed either at 48hh (a), 72 (b), 96h (c) or $120 \mathrm{~h}$ (d) and imaged by confocal microscopy. BRA and SOX2 proteins are stained in red and white, respectively. VENUS signal (green) reports Gata6 expression and Hoechst (Blue) marks the nuclei. Gastruloids corresponding to the 3D renderings shown in Fig. 1a. Each fluorescent channel is displayed to the right of each merged image. Gata6 or Gata6 and SOX2 signals were undetectable in $\mathbf{a}$ and $\mathbf{b}$, respectively, and therefore not shown. Three z-sections are shown for each gastruloid. The bright field outline of each gastruloid is indicated by the dashed lines. Scale-bars as indicated.

Extended data Figure 2. a. Heat map showing the temporal evolution of 97 out of the 250 most variable genes throughout embryonic development from E6.5 to E9.5 (left) and their corresponding expression over the gastruloid time-course, from $24 \mathrm{~h}$ to $168 \mathrm{~h}$ (right). Expression levels are highlighted by color scale from blue to red (bottom left). Genes were clustered according to their 
expression behaviour in the embryo and enriched GO term categories were identified for each cluster by using the Gorilla and Revigo tools (see supplementary material and methods and Supplementary file 1). Finally, a functional classification of each cluster was established based on the identified GO term categories and literature-based evidences. b. Expression of markers for different embryonic tissues through the gastruloid time-course. The two replicates of each time point are represented by triangles and circles. The black dotted line in each plot represents the average behaviour of the different genes displayed in the plot. c. PCA analysis of RNAseq datasets from either pooled or individual gastruloids using the top 1000 most highly expressed genes. Despite different strategies used for pooled versus individual gastruloids RNAseq (accounting for the sample segregation across PC1), the clustering of pooled and single-gastruloid datasets illustrates both the homogeneity of gastruloid cultures and the representativeness of pooled samples to single gastruloid samples.

\section{Extended data Figure 3: Gastruloids display spatio-temporal organization in the expression} profiles of neural, mesodermal and endodermal marker genes. a-f. The expression profiles of several genes normally expressed in the embryonic neural, mesodermal and endodermal domains was analyzed by plotting the RNAseq data in pooled gastruloids in heatmaps of scaled gene expression (a, c, e) and/or by WISH (b, d, f). a-b. Expression of different neural markers was detected in our RNAseq. Genes like Lnfg or Irx 3 were detected forming continuous and homogenous domains located in the central and dorsal portion of the gastruloids, reminiscent of their expression domains in the embryo (b, upper panels). Instead, genes involved in notch signaling in neural progenitors (Hes5, Dll1) and in the terminal differentiation of neural precursor (Phox2a, Mnx1) displayed a salt and pepper expression pattern, consistent with the lack of an organized neural tube structure (Extended data Figs. 4-5). However, the latter mRNAs also displayed a graded distribution along the anterior to posterior extension of the gastruloid axis and were absent from its posterior half (empty red arrowheads). c-d. Genes normally expressed in different types of mesoderm precursors in the embryo (e.g. Tcfl5 in paraxial somatic mesoderm, Osrl in intermediate mesoderm, Bra in notochord and PSM and Pecam in lateral plate mesoderm) were expressed in reproducible and spatially restricted domains within the gastruloids. e-f. Endoderm specific genes were also expressed in gastruloids. Particularly, genes expressed in the embryonic digestive tract were consistently found on the ventral side of gastruloids. For each gene, the proportion of gastruloids displaying the reported expression pattern is shown in the upper right corner of the image, expressed as a fraction of the total number. Scale bar: $100 \mu \mathrm{m}$. 
Extended data Figure 4. a. Gastruloids formed from Soxl ${ }^{G F P}$ (green); Bra ${ }^{\text {mCherry }}$ (red) mESCs were fixed at 168h AA and stained for SOX2. White arrowheads indicate tubular SOX2/SOX1 positive neural structures. Red arrowheads point to the presumptive digestive tube. b. Gastruloids at $144 \mathrm{~h}$ were WISHed for Sox 2 and Meox 1 antisense probes, cryo-sectioned in $8 \mu \mathrm{m}$ thick transversal crosssections and counter-stained with Nuclear Fast Red. Sox2 positive cells localized predominately in a compact dorsal domain, whereas Meoxl signals was found in two bilateral domains. The domain of expression of each gene is delimited with white dashed lines. c. Haematoxylin-Eosin staining of transversal paraffin sections of different gastruloids at 120h AA, showing the cell type diversity and several degrees of tissue organization. d. Gastruloids formed from Sox ${ }^{G F P}$ (green); Bra ${ }^{\text {mCherry }}$ (red) mESCs were fixed and stained at $168 \mathrm{~h}$ for OLIG2 (top-panel, white), PAX3 (mid-panel, red) and PAX7 (lower-panel, red). Scale bars as indicated. c, d. Gastruloids formed from Sox ${ }^{\text {GFP }}$ (green); $\mathrm{Bra}^{m \text { Cherry }}$ (red) mESCs collected at $168 \mathrm{~h} \mathrm{AA}$ and stained for SOX17 (magenta in c) or CDX2 (magenta in d). Scale bars as indicated.

Extended data Figure 5. a-e. Double FISH staining of gastruloids from Soxl ${ }^{\text {GFP }}$ (green); Bra ${ }^{\text {mChrry }}$ (red) mESCs at 144h with Meox1 and Cyp26a1 (a), Sox2 and Shh(b), Sox2 and Meox1 (c), Meox1 and Hoxd4 (d) or Sox2 and Hoxd4 (e). Scale bar: $200 \mu \mathrm{m}$

Extended data Figure 6. a-b. Gastruloids formed from Nodal $^{Y F P}$ mESCs were fixed at $120 \mathrm{~h} \mathrm{AA}$. They were stained for CDX2, YFP (Nodal ${ }^{Y F P}$ ) and E-Cadherin (a, top panel), CDX2, YFP (Nodal ${ }^{Y F P}$; green) and Phalloidin (a, bottom panel) or CDX2, YFP and E-CADHERIN (both with an Alexa-488 secondary antibody) and SOX2 (b). Maximum intensity projection of a representative gastruloid in $\mathbf{b}$, with the node-like structure highlighted. Hoechst marks the nuclei (greyscale in a, blue in b). c-d. In situ hybridizations of gastruloids at different time-points AA. Asterisk in d mark the presumptive node-like cells. White arrowheads point towards Nodal expressing cells distributed asymmetrically, on the lateral side of the gastruloid. e. Three dimensional renderings of confocal stacks of $120 \mathrm{~h}$ gastruloids containing a Nodal ${ }^{Y F P}$ reporter gene (green) and stained for SOX2 (white) and BRA (red) proteins. SOX2 signal identifies dorsal cells. Left and right panels show the same gastruloid, imaged from two different polar directions i.e. top (dorsal) and bottom (ventral) or 'left' and 'right' depending on the orientation of the gastruloid. Insets in specific panels show a cross-section through the gastruloid at the indicated $z$ plane. White arrowheads indicate the region of biased Nodal expression. Empty white arrowheads point to the node-like cells marked by the $N_{0}{ }^{Y F P}$ reporter gene. See also Fig. 4d for and Extended data Table 1. f. In situ hybridizations of gastruloids $120 \mathrm{~h}$ (left) and $144 \mathrm{~h} \mathrm{AA}$ (right). The midline of the gastruloid is marked by a dashed white line. White 
arrowheads point towards Nodal expressing cells distributed asymetrically on the lateral side of the gastruloid. In $\mathbf{c}, \mathbf{d}$, and $\mathbf{f}$, the proportion of gastruloids displaying the reported expression pattern is shown in the bottom left corner of each image, expressed as a fraction of the total number.

Extended data Figure 7. a, b. Dorsal (a) and ventral (b) sections of the same representative gastruloid shown in the 3D renderings in Fig. 4d, fixed at $72 \mathrm{~h} \mathrm{AA}$ and stained at $120 \mathrm{~h}$ for Nodal ${ }^{Y F P}$ (green), BRA (red) and SOX2 (white). Hoechst was used to mark the nuclei. Scale bar indicates $100 \mu \mathrm{m}$.

Extended data Figure 8. a. Heatmap of unscaled gene expression in E6.5-E9.5 mouse embryos, showing Hox gene transcript levels over time. b. RNAseq mapping showing Hoxa and Hoxd gene expression in these embryos. After a first wave of transcription of 5' Hoxa and Hoxd genes, likely reflecting their activation in extraembryonic tissues, the HoxA and $\operatorname{HoxD}$ clusters were progressively transcribed from E7.8 until E9.5 when expression of Hox13 paralogs was detected. c. Heatmap of unscaled gene expression in pooled gastruloids, showing Hox gene transcript levels over time. d. RNAseq mapping showing Hoxd gene expression in pooled gastruloids at different time points. Hoxd genes sub-groups are progressively activated starting at $72 \mathrm{~h}$ until $168 \mathrm{~h}$ AA, when expression of Hoxd13 starts to be detected (e), thus resembling the temporal activation described in vivo (a, b). e. Whole mount in situ hybridization of gastruloids collected at different time points and showing the detectable initiation of different Hoxd genes expression. Each panel report the earliest stage where transcripts of the corresponding gene were detected (black arrowhead). Expression of Hoxd4 was already strong at $96 \mathrm{~h}$ AA indicating that its transcripts are rapidly upregulated compared to Hoxd 9 which is faintly expressed at this stage. Scale bar: $100 \mu \mathrm{m}$.

Extended data Figure 9. a. Principal component analysis (PCA) based on Hox transcripts datasets only, extracted from individually sequenced gastruloids across time points (10 individual organoids per time point). The analysis was carried out using the $\log _{2}$ transformed FPKM+1 value of all 39 Hox genes. Replicate batches of organoids primarily cluster according to their age at collection. The clustering revealed the low sample-to-sample variation. Instead, replicates were clearly separated through the temporal parameter, representing $93.6 \%$ of total sample variation. b. Comparison of Hoxa (top panel) and Hoxd (bottom panel) gene expression profiles amongst individual gastruloids confirmed the low inter-sample variation among time-points, illustrated with the $120 \mathrm{~h}$ condition. c. Whole mount in situ hybridization of $168 \mathrm{~h}$ AA gastruloids showing the expression of different Hoxa 
paralogs. The proportion of gastruloids displaying the reported expression pattern is shown in the upper right corner of the image, expressed as a fraction of the total number. Scale bar: $100 \mu \mathrm{m}$.

Extended data Figure 10. a. Dot plot representing the progression in the measured longitudinal extension of gastruloids produced either from ES or from iPS cells. b. Light microscopy images showing representative examples of gastruloids at the different time-points analysed in (a). Zoom: 10x. Note that iPS derived gastruloid display delay in their longitudinal extension rate and at $120 \mathrm{~h}$ AA, they are significantly smaller than their ESC-derived counterparts. For this analysis, gastruloids were produced starting from the same number of cells (800 cells per well). c. Dot plots representing the mRNA levels of Bra, showing comparable dynamics of this gene in both types of gastruloids. d. Confocal images showing the expression of Oct4, SOX2 and BRA (upper panel) or of Oct4, SOX1 and CDX2 (bottom panel) in 120h gastruloids derived from the iPS cell line Oct4::Gfp (IpSL40N). iPS-derived gastruloids were fixed at 120h AA and stained for SOX2-BRA (left) and CDX2SOX1 (right). Oct4::GFP signal is shown in greys. Scale bars: $200 \mu \mathrm{m}$. e. Dot plots representing the mRNA levels of Hoxd genes in ES or iPS cell-derived gastruloids collected at different timepoints AA. Both gastruloids sequentially activated Hoxd gene expression. However, their temporal activation seemed to be delayed in iPS gastruloids (especially that of the most 3' Hoxd paralogs). e. Whole mount in situ hybridization of 144h AA gastruloids, showing the expression of different Hoxd paralogs. Even though iPS derived gastruloids reproduced the antero-posterior Hoxd collinear expression, Hoxd9 expression domain often extended more anteriorly when compared to ESs cell derived gastruloids (see Fig. 5e), occupying approximately the same domain than Hoxd4. Also patches of Hoxd negative cells were often found within the Hoxd4/Hoxd9 expression domain (white asterisks). Scale bar: $100 \mu \mathrm{m}$.

Supplemental Movies 1 and 2 (corresponding to Fig. 1a 96h and 120h AA). a. Gastruloids were produced from Gata6 ${ }^{\text {H2B-Venus }} \mathrm{mESC}$ s treated with Chiron between $48 \mathrm{~h}$ and $72 \mathrm{~h} \mathrm{AA}$, fixed and stained at either 96h AA (movie 1) or 120h (movie 2) for Gata6 (green), BRA (red) and SOX2 (white) and imaged by confocal microscopy as indicated in Materials and Methods. Each fluorescent channel is highlighted in turn for clarity. Hoechst was used to stain the nuclei.

Supplemental Movies 3 and 4 (for Fig. 3d). Gastruloids were produced from Nodal ${ }^{Y F P}$ mESCs and treated with Chiron between $48 \mathrm{~h}$ and $72 \mathrm{~h} \mathrm{AA}$, fixed and stained at $120 \mathrm{~h}$ for YFP (green), BRA (red) and SOX2 (white). Views from the dorsal (movie 3) and ventral (movie 4) aspects are shown. The Node-like region and bilateral expression of Nodal is highlighted in the ventral region (movie 4). 


\section{References}

1. Rossant, J. \& Joyner, A. L. Towards a molecular-genetic analysis of mammalian development. Trends Genet 5, 277-83 (1989).

2. Simunovic, M. \& Brivanlou, A. H. Embryoids, organoids and gastruloids: new approaches to understanding embryogenesis. Development 144, 976-985 (2017).

3. van den Brink, S. C. et al. Symmetry breaking, germ layer specification and axial organisation in aggregates of mouse embryonic stem cells. Development 141, 4231-42 (2014).

4. Sasai, Y., Eiraku, M. \& Suga, H. In vitro organogenesis in three dimensions: self-organising stem cells. Development 139, 4111-21 (2012).

5. Turner, D. A., Baillie-Johnson, P. \& Martinez Arias, A. Organoids and the genetically encoded self-assembly of embryonic stem cells. Bioessays 38, 181-91 (2016).

6. Turner, D. A. et al. Anteroposterior polarity and elongation in the absence of extraembryonic tissues and spatially localised signalling in Gastruloids, mammalian embryonic organoids. Development (2017). doi:10.1242/dev.150391

7. Zhao, R. et al. Loss of both GATA4 and GATA6 blocks cardiac myocyte differentiation and results in acardia in mice. Dev Biol 317, 614-9 (2008).

8. Kurokawa, D. et al. Regulation of Otx2 expression and its functions in mouse forebrain and midbrain. Development 131, 3319-31 (2004).

9. Carninci, P. et al. The transcriptional landscape of the mammalian genome. Science 309, $1559-63(2005)$.

10. Wang, P. et al. A molecular signature for purified definitive endoderm guides differentiation and isolation of endoderm from mouse and human embryonic stem cells. Stem Cells Dev. 21, 2273-2287 (2012). 
11. Beck, F., Erler, T., Russell, A. \& James, R. Expression of Cdx-2 in the mouse embryo and placenta: possible role in patterning of the extra-embryonic membranes. Dev Dyn 204, 219-27 (1995).

12. Blum, M. et al. Gastrulation in the mouse: the role of the homeobox gene goosecoid. Cell 69, $1097-1106(1992)$.

13. Deschamps, J. \& van Nes, J. Developmental regulation of the Hox genes during axial morphogenesis in the mouse. Development 132, 2931-42 (2005).

14. Deschamps, J. \& Duboule, D. Embryonic timing, axial stem cells, chromatin dynamics, and the Hox clock. Genes Dev 31, 1406-1416 (2017).

15. Scotti, M. \& Kmita, M. Recruitment of 5' Hoxa genes in the allantois is essential for proper extra-embryonic function in placental mammals. Development 139, 731-9 (2012).

16. Harrison, S. E., Sozen, B., Christodoulou, N., Kyprianou, C. \& Zernicka-Goetz, M. Assembly of embryonic and extraembryonic stem cells to mimic embryogenesis in vitro. Science 356, (2017).

17. Kadoshima, T. et al. Self-organization of axial polarity, inside-out layer pattern, and speciesspecific progenitor dynamics in human ES cell-derived neocortex. Proc Natl Acad Sci U A 110, 20284-9 (2013).

18. Lancaster, M. A. et al. Cerebral organoids model human brain development and microcephaly. Nature 501, 373-9 (2013).

19. Rivron, N. C. et al. Blastocyst-like structures generated solely from stem cells. Nature 557, 106-111 (2018).

20. Heisenberg, C. P. \& Bellaiche, Y. Forces in tissue morphogenesis and patterning. Cell 153, 948-62 (2013).

21. Guerreiro, I. et al. Reorganisation of Hoxd regulatory landscapes during the evolution of a snake-like body plan. Elife 5, (2016). 
a

Oh $24 \mathrm{~h} 48 \mathrm{~h} 72 \mathrm{~h}$ 96h 120h $144 \mathrm{~h} 168 \mathrm{r}$

b

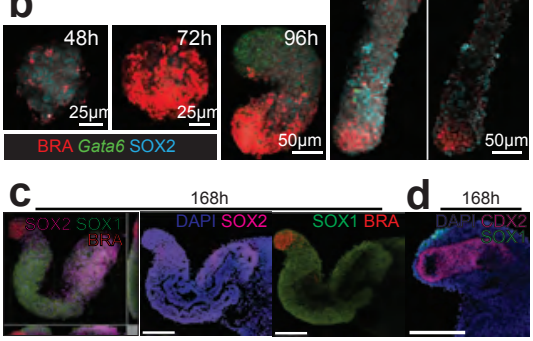

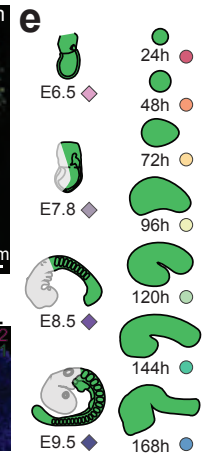

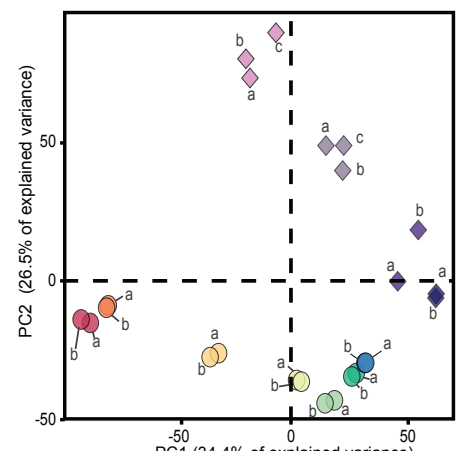

Figure 1 


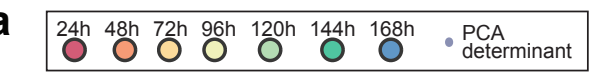

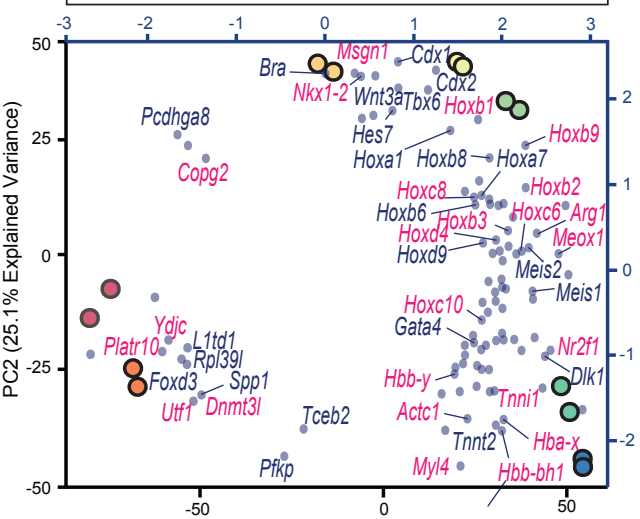

b Gastruloids

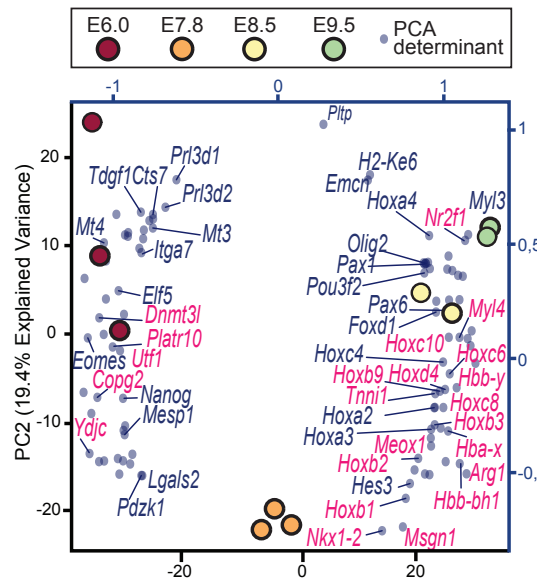

PC1 (53.7\% Explained Variance)

PC1 (55.9\% Explained Variance)

Embryos $\quad \log 2(\mathrm{FPKM}+1) \mathbf{0} \quad \begin{array}{lll}\mathbf{2} & 2\end{array}$

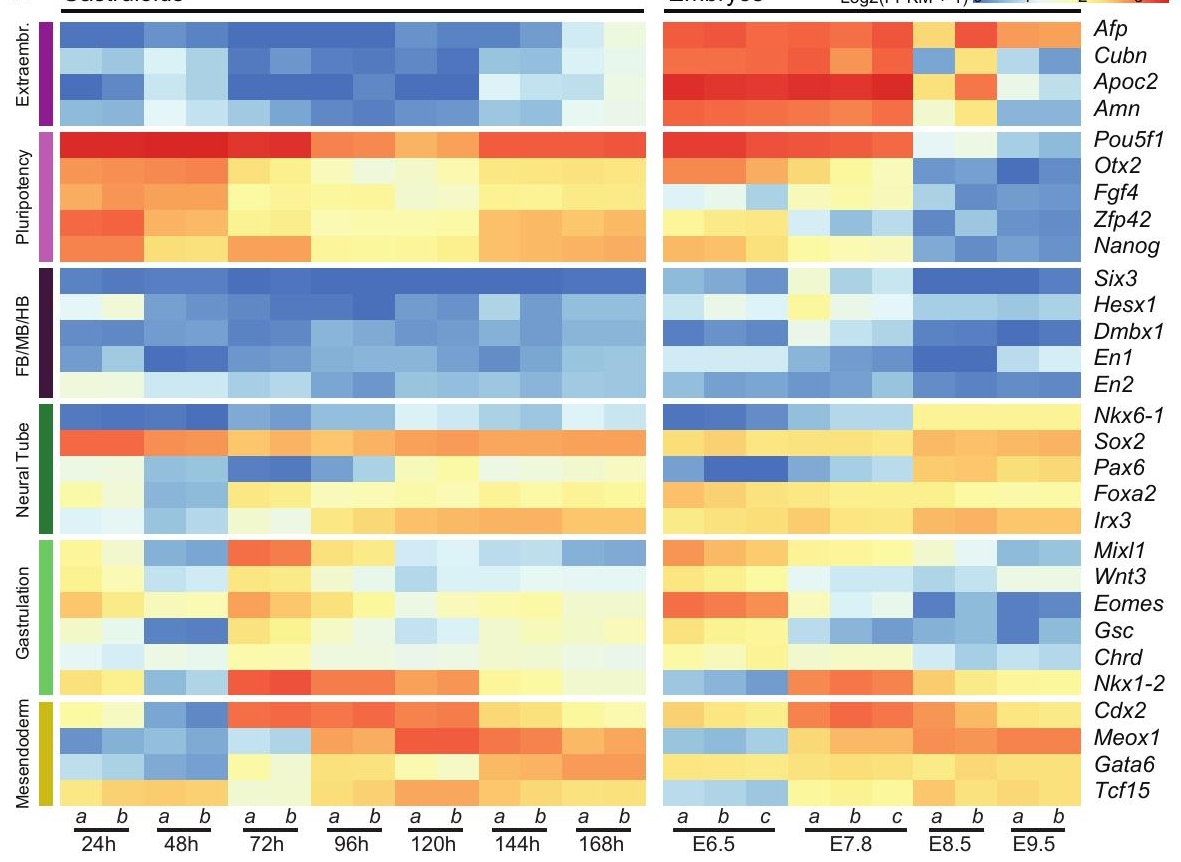




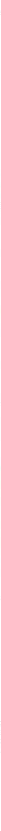

Figure 3 

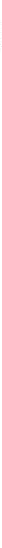

C

Hoxd4 Hoxd9 Hoxd11 Hoxd13
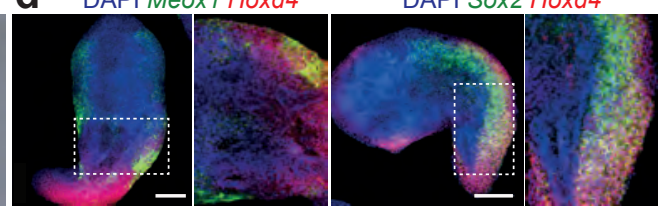

Figure 4 
a Hoechst/BRA/

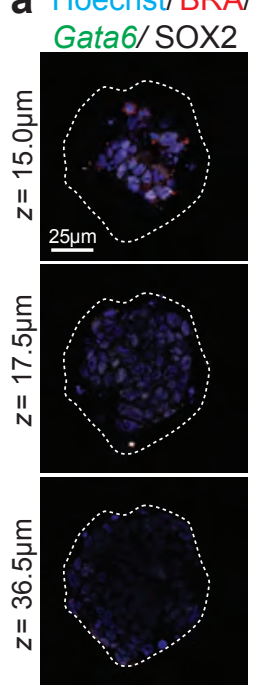

Gata6

BRA

sox2

b Hoechst/BRA/

Gata6

BRA

sox2

c Hoechst/BRA/
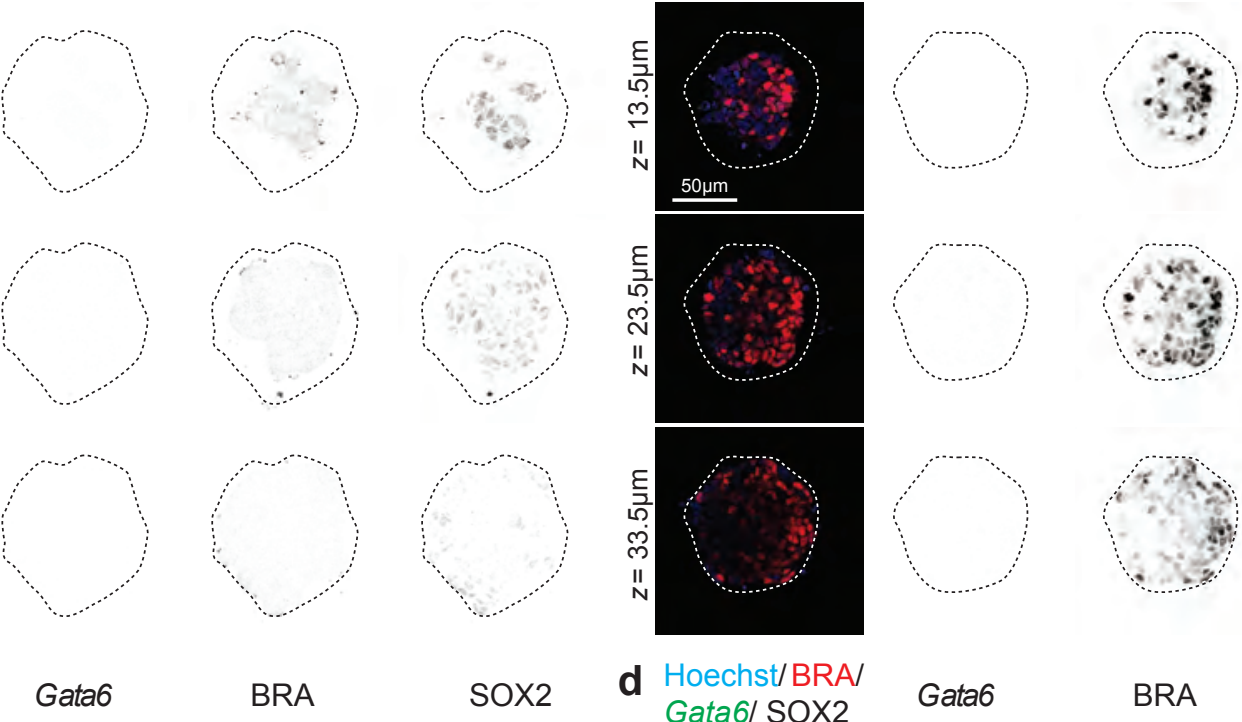

d Hoechst/BRA/
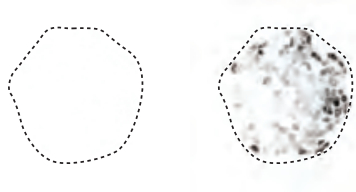
Gata6/ SOX2
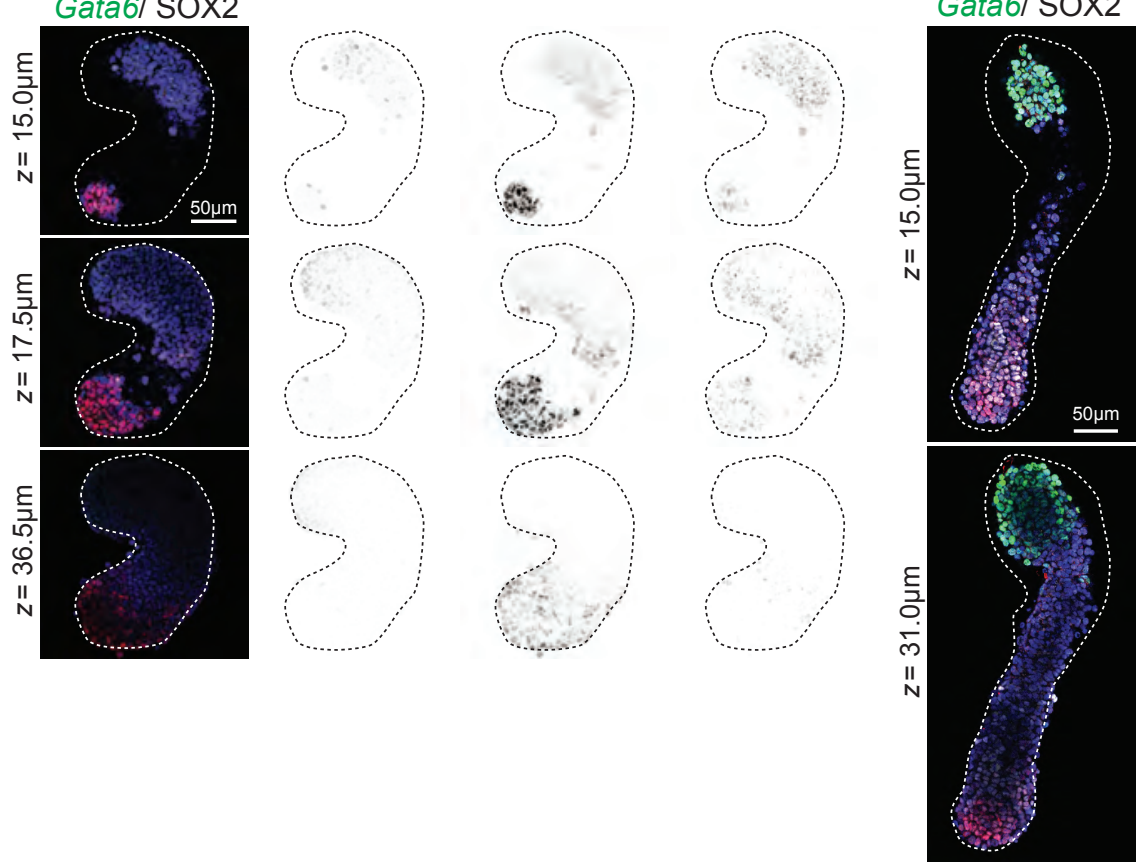

Gata6

BRA

sox2
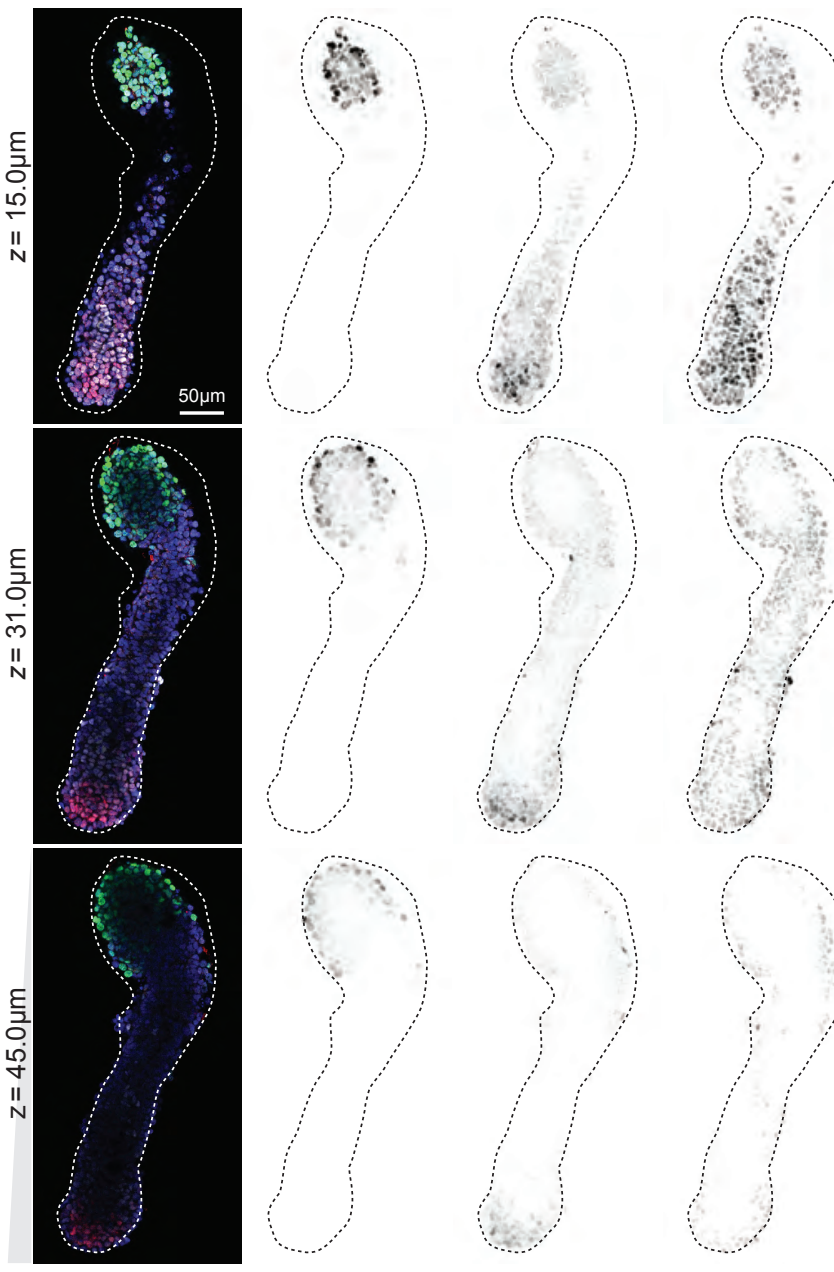

.
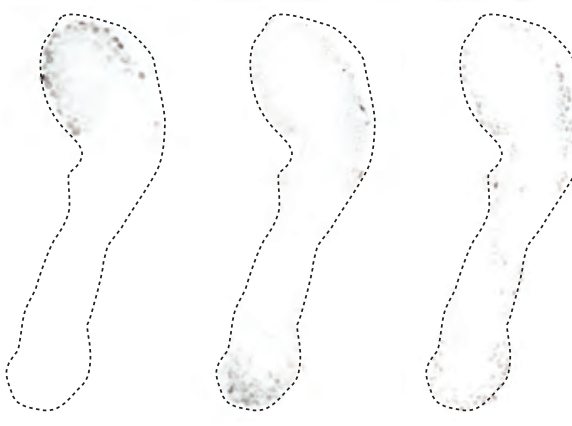

\section{Extended data Figure 1}


a

a

Embryo

Gastruloids $\log _{2}(\mathrm{FPKM}+1)$

b

Epiblast
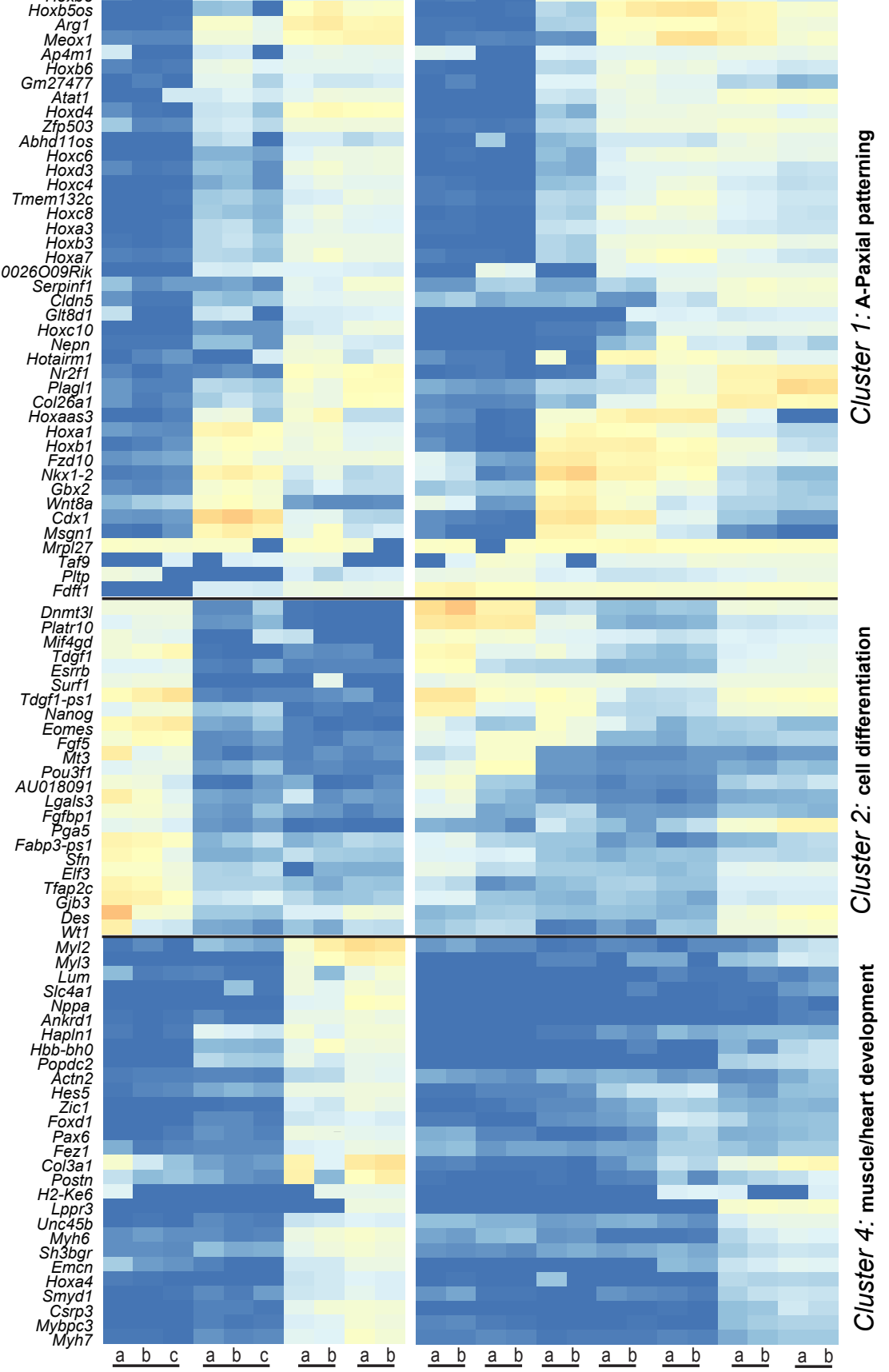

$\frac{a b c}{E 6.5} \quad \frac{a b c}{E 7.8} \quad \frac{a b}{E 8.5} \frac{a}{E 9.5} \quad \frac{a b}{24 h} \quad \frac{a}{48 h} \quad \frac{a}{72 h} \quad \frac{a}{96 h} \quad \frac{a b}{120 h} \quad \frac{a}{144 h} \quad \frac{a b}{168 h}$

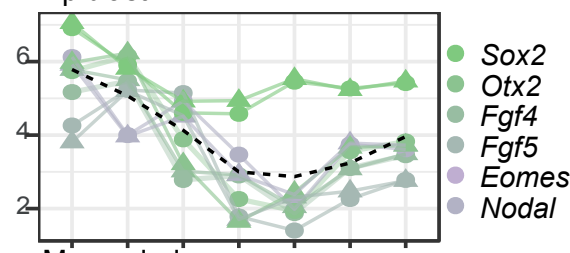

Mesendoderm

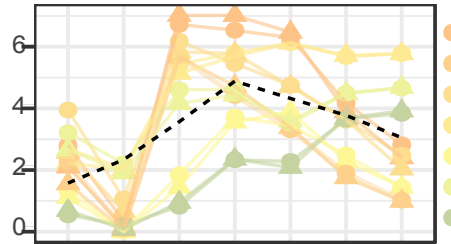

$C d x 2$

Evx1

Fgf8

Cdh2

Cer1

Snai1

Gastrulation

Snai2

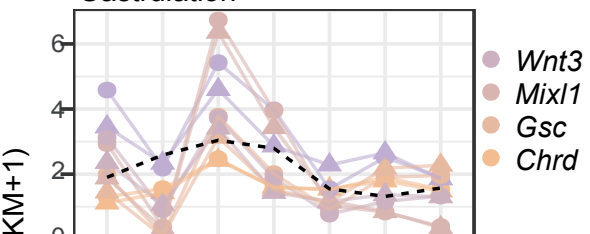

antermediate Mesoderm

Oै $6=0 \begin{aligned} & \text { Osr1 } \\ & \text { Hnf1b }\end{aligned}$

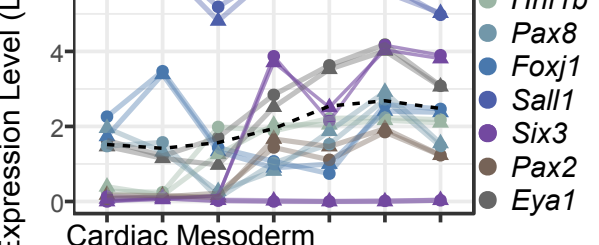

Cardiac Mesoderm

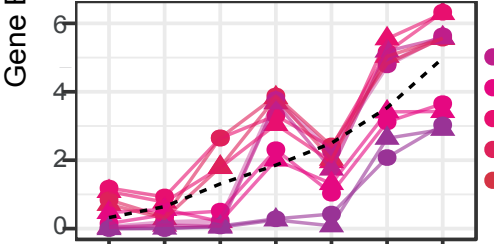

- Hand2

- Is/1

Gata4

- Gata6

Actc1

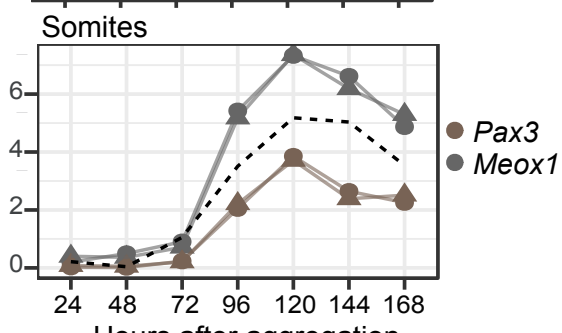

Hours after aggregation

10

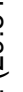

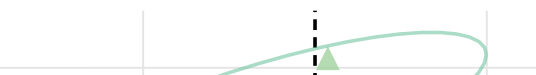

$1 .$.

$\begin{array}{lcc}-25 & 0 & 25 \\ \text { PC1 } & \text { (54.5\% } & \text { Explained variance) }\end{array}$

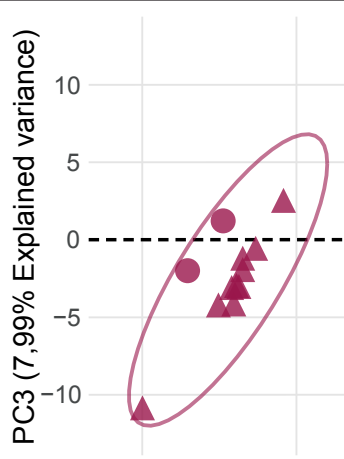

$-20$

$-10$

PC2 (29.84\% Explained variance) 


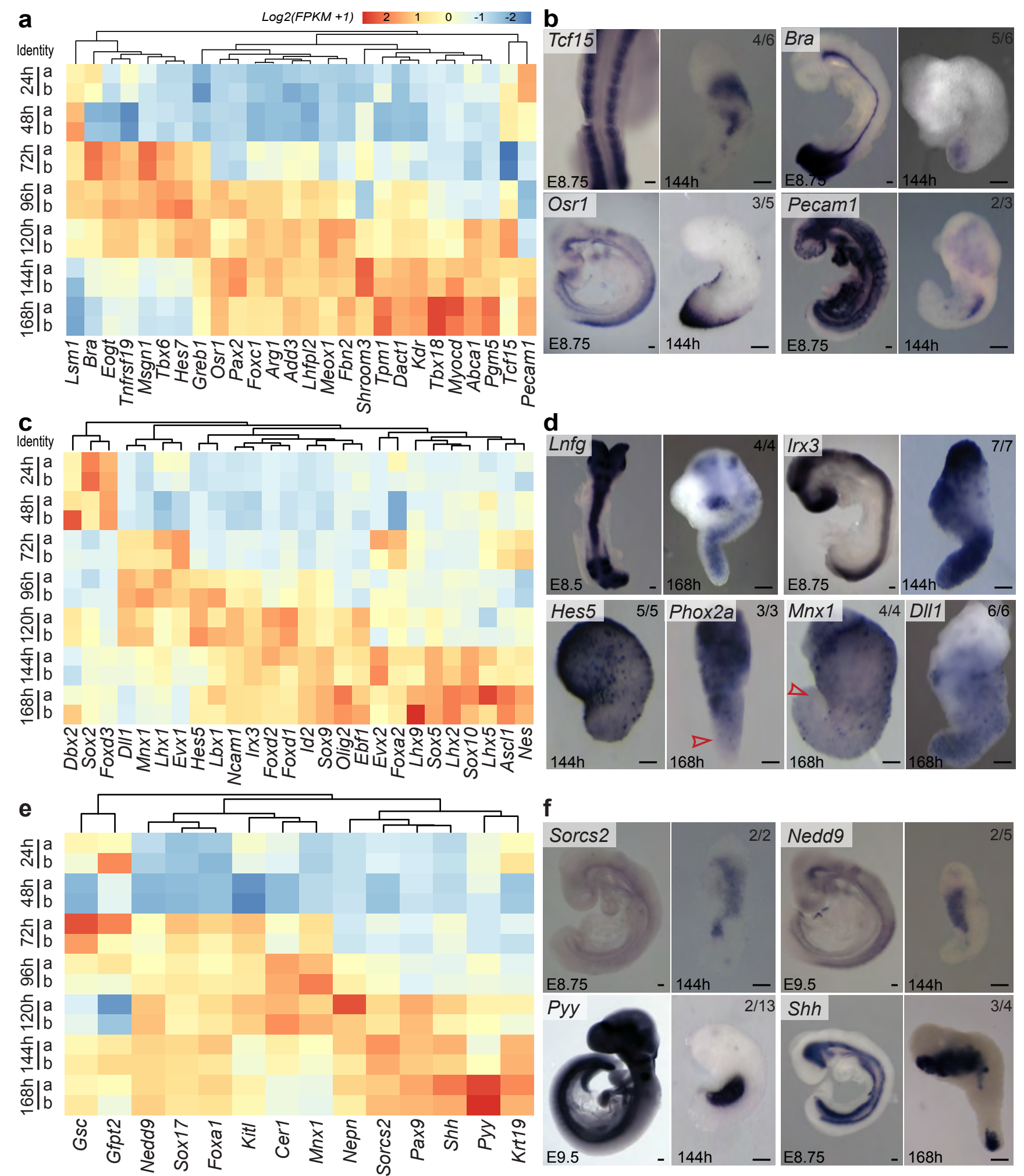

Extended data Figure 3 


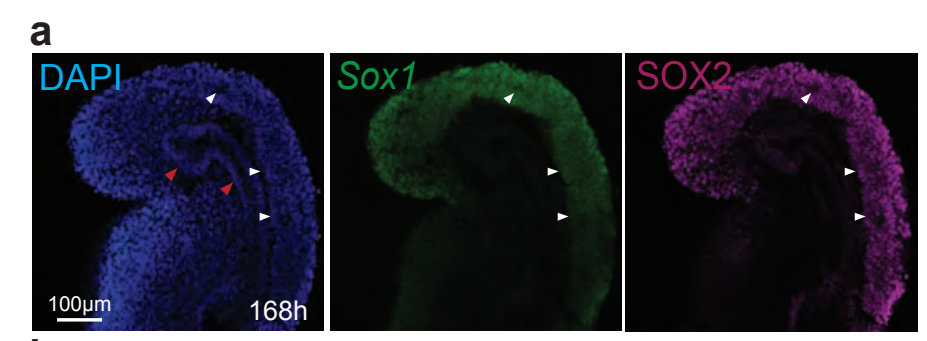

b

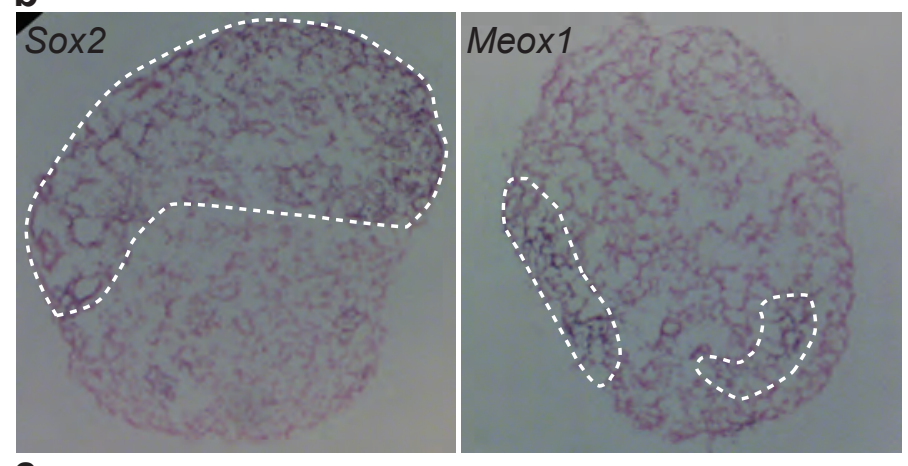

C
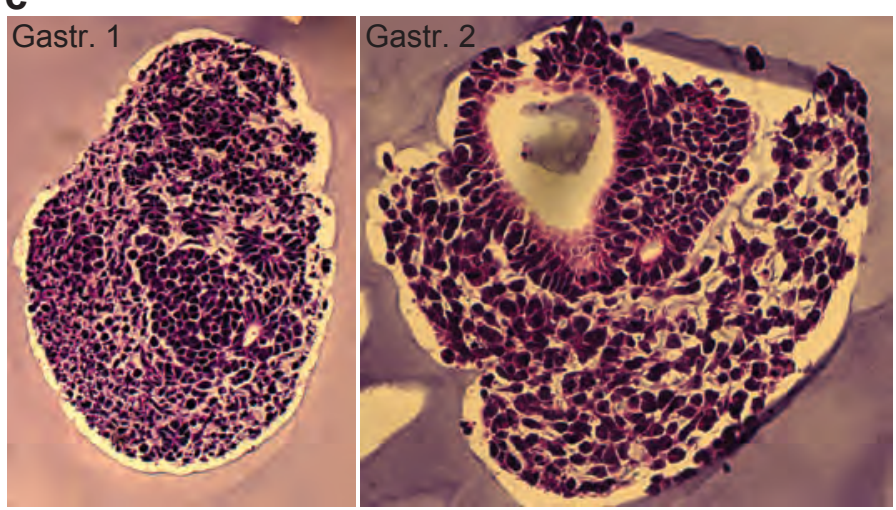

d
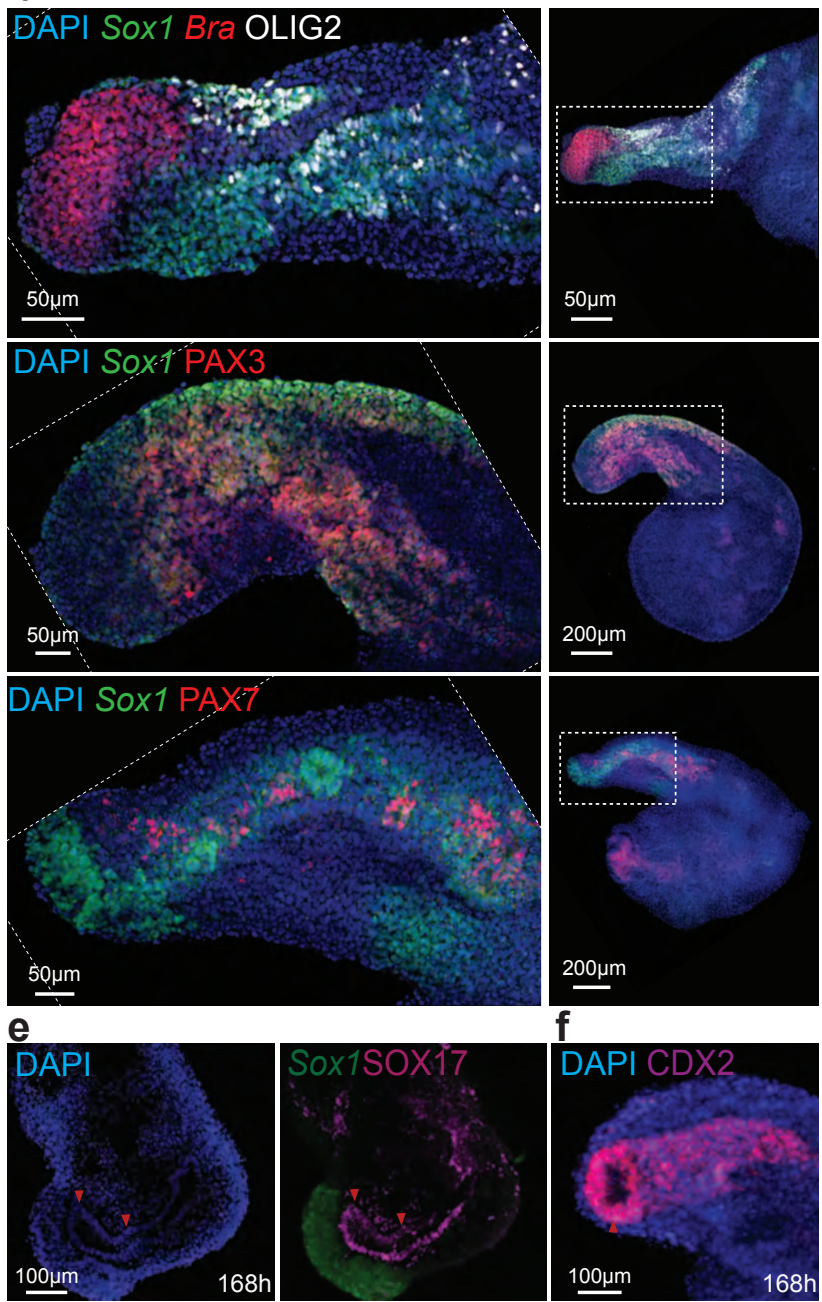

$200 \mu \mathrm{m}$
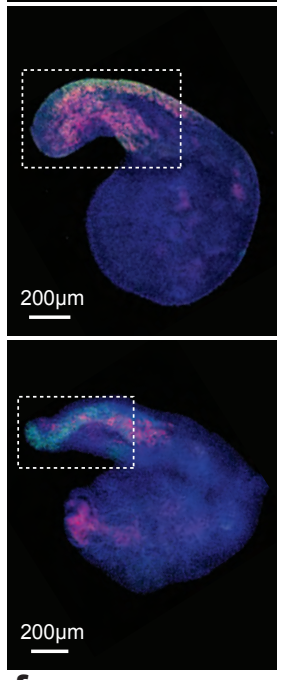

f

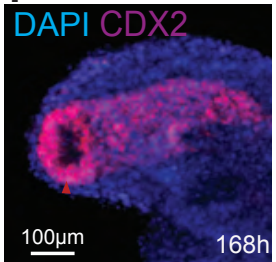

Extended data Figure 4 


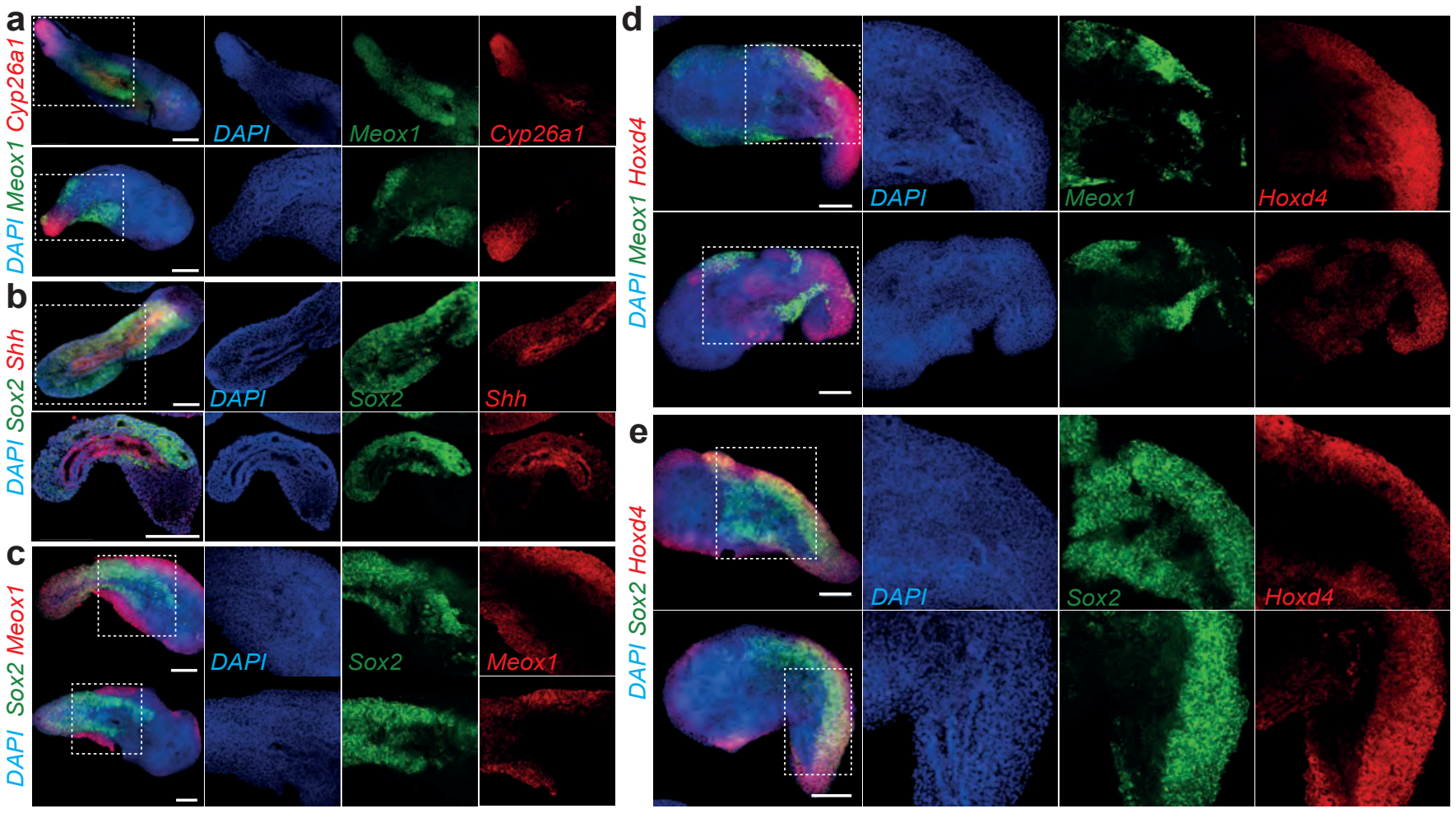

Extended data Figure 5 

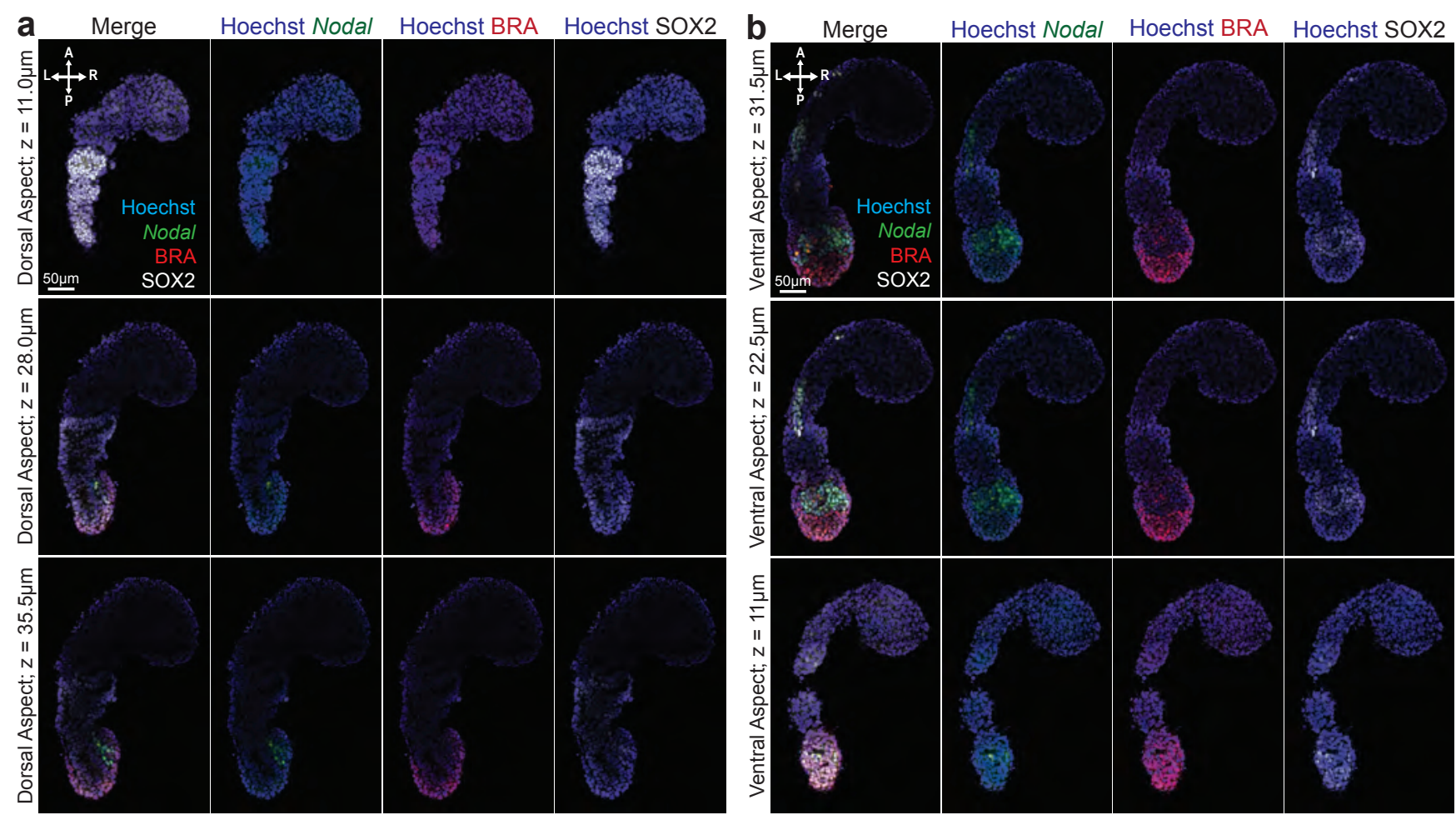

Extended data Figure 7 


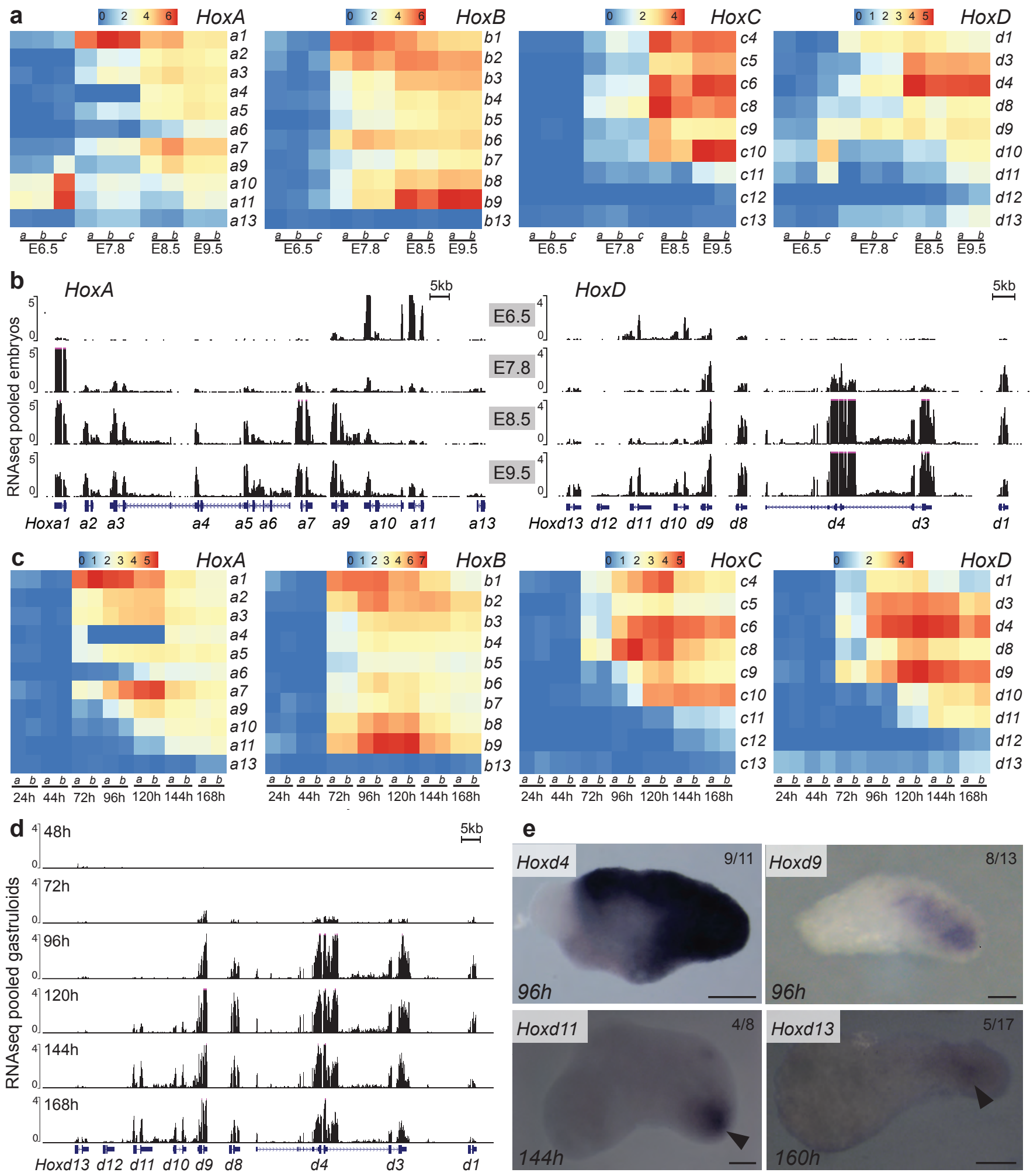

Extended data Figure 8 


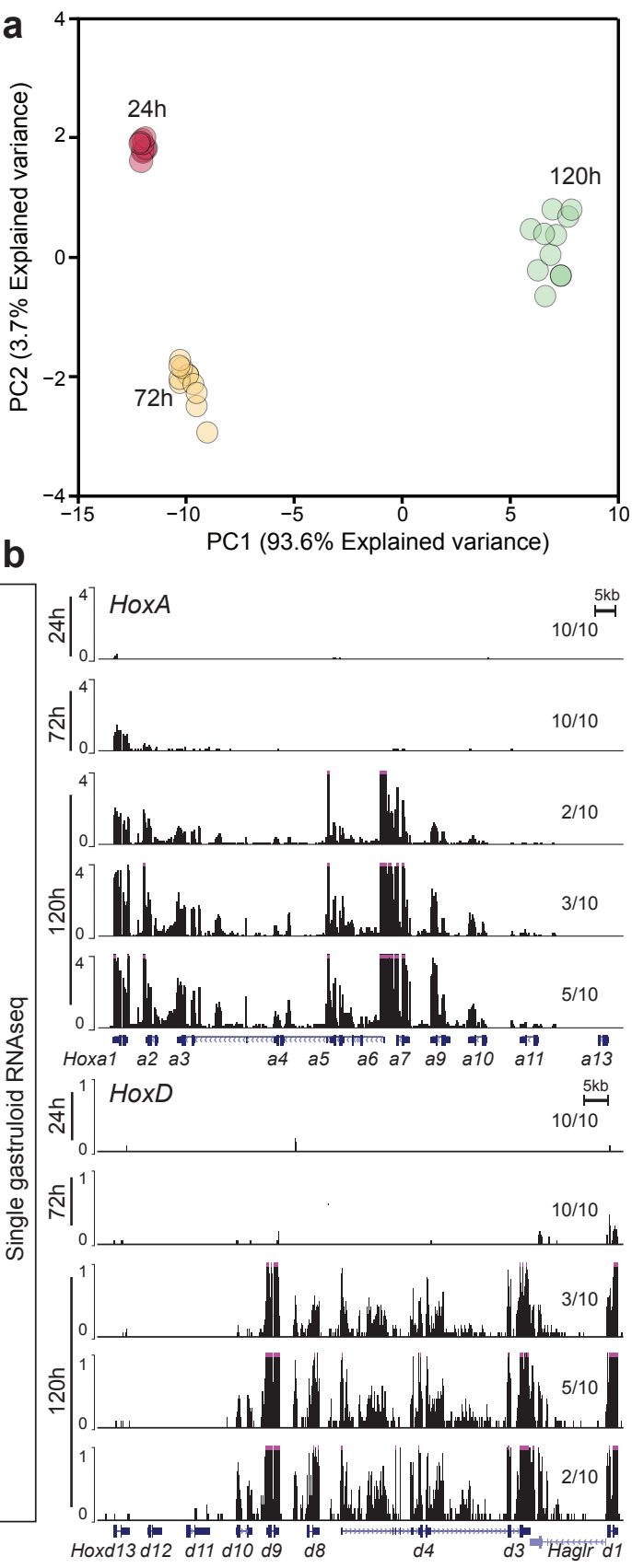

c

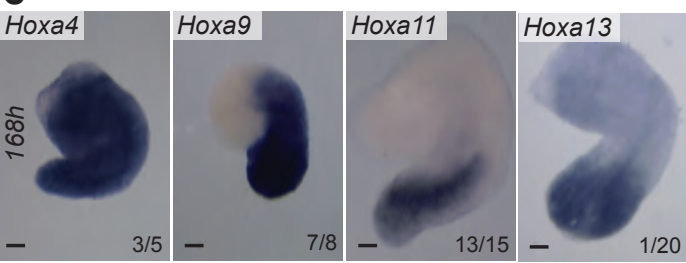

Extended data Figure 9 